\title{
Stability and Change: \\ Illustrations with Categorical and Binary Responses
}

\author{
Fernando Rajulton \\ Zenaida R. Ravanera \\ Population Studies Centre \\ University of Western Ontario
}

\begin{abstract}
Longitudinal data consist of time-sequences of measurements, counts or categorical responses from the same experimental units. Thus, they have a distinct advantage over cross-sectional data in that they provide us the information on both stability and change. It is recommended therefore that any longitudinal study should tap this information through available techniques. In social science research, the use of categorical and binary responses is more frequent than the use of continuous-time responses. This paper aims to show that more detailed and sophisticated analysis can be done even with categorical and binary sequences collected through longitudinal surveys. After proposing two paradigms that may be used in the explanations of stability and change, the paper presents two illustrations for the analysis of categorical and binary sequences.
\end{abstract}


Empirical Research and Applications -

Fernando Rajulton and Zenaida Ravanera

\section{Résumé}

Les données longitudinales se composent de mesures ordonnées dans le temps, soit des comptes ou des réponses catégoriques à partir des mêmes unités d'expérience. Ainsi, elles ont un avantage distinct par rapport aux données transversaux du fait $q u=e l l e s$ nous fournissent les informations sur la stabilité et le changement. On recommande donc que n'importe quelle étude longitudinale devrait inclure cette information par des techniques disponibles. Dans la recherche en sciences humaines, l'utilisation des réponses catégoriques et binaires est plus fréquente que l'utilisation des réponses continues dans le temps. Cet article vise à démontrer qu'une analyse plus détaillée et plus sophistiquée peut être faite même avec des données catégoriques et binaires ordonnées dans le temps. Après avoir proposer deux paradigmes qui peuvent être utilisés dans les explications de la stabilité et du changement, le papier présente deux illustrations pour l'analyse des données ordonnées, soit catégoriques ou binaires.

Key Words: Stability and change, binary sequences, unobserved heterogeneity, initial conditions, turnover tables

\section{Introduction}

Longitudinal data consist of time-sequences of measurements, counts or categorical responses from the same (experimental) units. Thus, they are closely related to time series data. Techniques used for time series data, however, may not adequately capture the rich information contained in longitudinal data. [Readers interested in time series approach to analysing longitudinal data can refer to Jones (1993) or Frederiksen and Rotondo (1979).] Besides the time series characteristics, longitudinal data have some important aspects that call for different techniques of analysis. Unlike time series data, longitudinal data have inherent state dependencies that can be examined more adequately only with stochastic frameworks.

In particular, the importance of initial conditions can never be overstated in analyzing longitudinal data. The initial (as well as final) conditions clearly vary with the research designs and can have marked effects on inferences made from such data. For many individuals in a study, the initial (and final) conditions generally depend on the starting (and ending) dates of the study (that is, in which status we start observing individuals or in which status we end our observation). These are usually beyond a researcher $=\mathrm{s}$ control and mostly dependent on the availability of funds! An example below illustrates the relevance of initial status even in multivariate contexts. 
Stability and Change: Illustrations with Categorical and Binary Responses

Assuming that each unit in the sample comes from an underlying population, that information on socioeconomic covariates is available, and that the response patterns vary with time, treatment or covariates, longitudinal data have three salient features: subject behaviour, time behaviour and covariate behaviour. Longitudinal studies therefore should necessarily unpack these three effects, no matter whether through a simple exploratory technique or through more sophisticated models that incorporate stochastic frameworks and structural parameters. Even a simple exploratory technique can unravel these effects more efficiently than sophisticated techniques that lead nowhere. It would be a big mistake to rush on to multivariate analysis without having adequately examined the nature of available data.

To bring out the distinct information on subject, time and covariate behaviours buried in longitudinal data, plots are useful, particularly those that can display all the data points over time; for example, Parallel Plots and Trellis Graphs (Cleveland, 1993). ${ }^{1}$ Not all statistical packages may be able to produce these plots, one may have to use packages such as S-Plus for a good explorative study. ${ }^{2}$ Trellis graphs have certain technical features such as "main effects ordering" that help us find hidden relationships in multivariate data sets and how two variables change with one or more "conditioning" variables.

\section{Stability and Change}

In an analysis of subject, time and covariate behaviours, two distinct concepts are essential: Stability and change. These two concepts together differentiate longitudinal data from other forms of data and offer a distinctive advantage of longitudinal studies over other studies. The important point is that these two concepts go together; it would be a mistake to concentrate only on stability or only on change. As far as change occurs in a systematic way, there will always be a stable pattern underneath. Change occurs not only at the individual level but also at the system level. All the changes that we have observed at the individual level since World War II have not taken place in a vacuum. Increased opportunities led to social mobility, which in turn led to changes in value systems and then to changes in individual behaviour. Despite all these changes, one has to admit that some structural continuities remain. As Engel and Reinecke (1996) point out, a distinct case in point is social inequality despite all the progress made in almost all fronts, be they social, economic or cultural.

How, then, to examine stability and change at the same time? As Coleman (1990) argues, explanations of system behaviour should be based on knowledge about its component parts below the system level. How much a system changes depends on how change takes place at a lower level, that is, at the individual level. There is no system change if there is no change at the individual level or if there are opposing individual changes that cancel one another out. And, any change at the individual level should necessarily be "diffused" to other individuals such that the system recognizes that a change has taken place and tries to adjust itself to that change. 
Empirical Research and Applications -

Fernando Rajulton and Zenaida Ravanera

Thus, stability and change can be viewed as mutually influencing forces of systems on individuals and individuals on systems. Hence, the importance of life course and/or two-way multilevel analysis is evident while working with longitudinal data.

Some societies are characterized more by change while others more by stability. More developed societies are good examples of the former while less developed ones are good examples of the latter. If a society is characterized more by change, then there is a greater need for longitudinal research to examine the processes of both change and stability.

In studying change and stability, researchers will have to deal with complexities arising from independent or dependent samples, missing information, measurement error, observed and unobserved heterogeneity. All these topics cannot be dealt with adequately in a paper like this, but other papers published in this Special Issue touch on these complexities. In the next section, two paradigms are discussed, paradigms that may be useful to explain both stability and change

\section{Two Useful Paradigms for Explaining Stability and Change}

\section{Planned Behaviour}

Since the main purpose of collecting longitudinal data is to follow the sociopsycho-economic development or behavior of individuals over time, the paradigm of planned behaviour (generally used by psychologists) may help explain behavioural changes and adaptation. Demographers have used this paradigm in many contexts although with different terminologies, starting from the explanations offered for the demographic transition.

A central idea in this paradigm is the individual's intent to behave in a specific way. Intents are assumed to capture the motivations underlying behaviour. Psychologists postulate three conceptually independent determinants of intent: 1) Attitude toward the behaviour which refers to the degree to which the person has a favourable or unfavourable evaluation of the behaviour; 2) A social factor called subjective norm which refers to the perceived social pressure to perform or not to perform the implied actions; and, 3) the degree of perceived behavioural control, which refers to the perceived ease or difficulty of performing the behaviour depending on the experiences of the individual as well as expected impediments, obstacles, costs and benefits.

These three determinants usually have a specific ordering: the more favourable the attitude, the greater the perceived behaviour control and the stronger will be an individual $=\mathrm{s}$ intent to perform. Similarly, the greater is the social pressure, the greater is the perceived control and the stronger the intent. And, the stronger the intent is, the more predictable is a given behaviour. These ideas are not foreign to demographers familiar with the three 'determinants' of contraceptive practice: 
Stability and Change: Illustrations with Categorical and Binary Responses

ready, willing and able (Coale, 1973; Lesthaeghe and Vanderhoeft, 1998).

Reinecke and Schmidt (1996) apply this paradigm of planned behavior to examining use of condoms in new sexual contacts in a study of AIDS-risk function, but in the context of handling missing values. In the experience of many researchers, missing data is one of the practical problems encountered in working with this paradigm (or with the 'ready-willing-able' paradigm of demographerssee for example the MA thesis of Mannan (1999)). In addition, longitudinal data bring in the extra dimension of stability and change; intents, norms and control can all vary over time or stay the same for all times. Insights into the circumstances that favour change or stability can enrich our understanding of causal mechanisms, particularly the mutual influence of each "determinant" on the other two.

\section{Life Course Paradigm}

Longitudinal data typically provide information on individuals' life courses encompassing various domains of interest. We are able to examine how individual lives are shaped by personal characteristics as well as by social environment - a clear departure from the emphasis on the former for so long. Featherman and Lerner (1985) envisage a 'developmental contextualism' that would enable a study of 'person-population' processes in the near future.

Cain (1964) used the term 'life course' to encompass anthropological, sociological, and psychological concepts of 'aging' or maturing through an expected sequence of social roles. According to him, the life course patterns in all domains of society (such as the family, the polity, economy and religion) are characterized by 'age status synchronization' whereby certain people follow different trajectories from others. The term (life course) is therefore used in reference to a sequence of socially defined events and roles that an individual experiences over time. This is in distinction from the term 'life cycle' that allows for events and roles that do not necessarily proceed in a socially defined sequence (Elder, 1975).

The concept of the life course helps in dealing simultaneously and adequately with the once-problematic age-period-cohort effects. Life course links them all together in one perspective: the distinctive historical and cultural events (= period) experienced by people who not only develop according to a biological clock (= age) but also socially share these experiences with their peers (= cohort). Giele and Elder (1998) suggest a framework consisting of four links that connect all these major elements of life course studies:

1) Location in time and place or cultural background (period aspect);

2) Linked lives or social integration (cohort aspect);

3) Human agency or individual goal orientation (age aspect); and,

4) Timing of life events or strategic adaptation (longitudinal aspect). 
Empirical Research and Applications -

Fernando Rajulton and Zenaida Ravanera

In their view, the fourth dimension, namely timing, integrates the previous three, namely historical, social and individual activities. Obviously, then, timing of life events becomes the cornerstone of all life course studies, and longitudinal designs are the primary methods for promoting such studies. Therefore, either through retrospective or prospective designs, data should be collected, whenever possible, on historical contexts, family, work and social settings, health, well-being, goals and life satisfaction, and major timings of events.

The life course paradigm is also a useful tool for examining stability and change not only in individual behaviour but also in structural behaviour. Both stability and change are integral parts of the life course, because as change occurs in a systematic way, it will make for an either stable or changing pattern. In post-war developed societies, for example, increased prosperity, changing values and everburgeoning individualization are indicators of endless change, and yet much structural continuity remains at the same time; for instance the stable structure of social inequality.

Change is the result of a tension between the programming by society and the individual's own choice (Kohli, 1986). Thus, the connection between micro-level ageing over the life course and macro-level institutional change can be observed through the changing life course patterns of successive cohorts. Some cohorts introduce an 'innovation' in the life course that usually departs from tradition and prefigures an institutionalization of that way of life so that it becomes the standard (Kohli, 1986). Such innovations, though first viewed as deviance, create new milestones for individuals living in changing times, and at the same time demand the adjustment or creation of social policies that will allow and institutionalize the new life patterns. Thus, changes in individual lives can, and often do, result in structural changes as well. We can think of changes in the roles of women, retirement age, transition to adulthood, family types, as typical examples.

In our opinion, the life course paradigm necessarily involves an application of stochastic frameworks and structural parameters. Interested readers can read the other papers appearing in this Special Issue. Here, we shall concentrate on examining stability and change using the most frequently available variables in social science, namely categorical and binary variables.

\section{Analysing Categorical Variables for Stability and Change}

With repeated measurements of some characteristics or status over time, we can have a simple cross-tabulation of data for one time against another. Such a table is called a turnover table since it essentially gives us the information on the flow between categories or statuses. For simplicity, let us consider two time points 1 and 2. The corresponding turnover table is one where response items are classified by earlier and later time points (Time 1 categories as rows, Time 2 categories as columns). An example ${ }^{3}$ is given in Table 1, which cross-classifies the observed 
Stability and Change: Illustrations with Categorical and Binary Responses

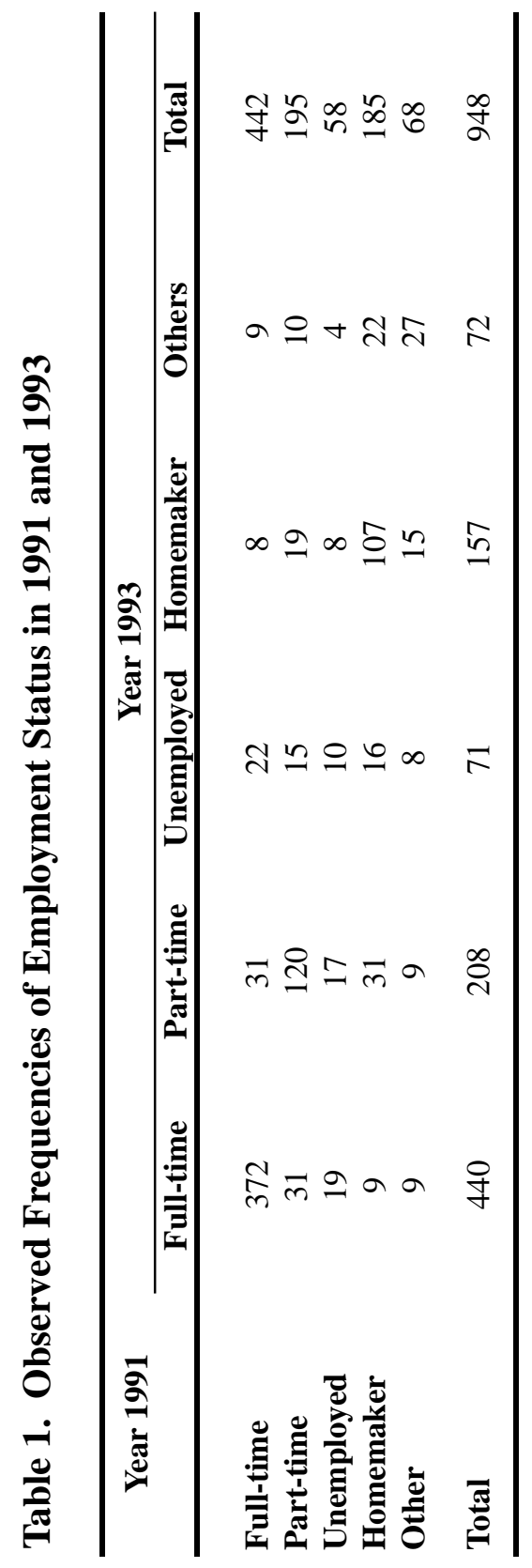


Empirical Research and Applications -

Fernando Rajulton and Zenaida Ravanera

frequencies of employment status of women living as single parent or two-parent families in London Ontario. The data are from a longitudinal study conducted by Avison (1996). During the first wave in 1991, 1020 interviews were completed and $91 \%$ of these respondents were successfully re-interviewed in the second wave in 1993. Even though a two-year gap is too short to observe large number of changes, this can still serve as an illustration for examining stability and change with such simple cross-tabulations. This analysis can lead to greater understanding of stability and change especially when data from more waves are also available.

As seen from the table, the majority (67\%) of women had the same employment status in both the years, while $33 \%$ changed their employment status. Of those who were working full-time in 1991,70 changed their status, moving mainly to parttime (31) and to unemployed (22) category. The marginal distributions suggest that women who were homemakers in 1991 experienced the largest net decline during the interval whereas the categories Part-time and Other had the greatest net increase.

Turnover Tables can be examined in different steps for stability and change implied in the data (see McCutcheon, 1996 for more details):

Step 1: As with the traditional contingency table analysis, we can test the assumption of independence using the $\chi^{2}$ statistic. The independence hypothesis assumes that employment status in 1991 is unrelated to that in 1993, in other words, there is no systematic relationship between statuses at time 1 and time 2 . Although this hypothesis can be examined using the traditional packages like SPSS and SAS, we shall use the package $l$ EM (log-linear and event history analysis with missing data) by Vermunt (1997) for the sake of using the same package for other types of analyses described in the following steps (where SPSS and other packages cannot be used). ${ }^{4} l$ EM uses the log-linear format and produces additional parameters that can be interpreted in a meaningful way. The results from $l \mathrm{EM}$ for test of independence are shown in Table 2 along with the commands used in the package $l E M$. [See the manual accompanying $l E M$ for these commands: 'man' stands for 'manifest variables,' 'dim' for dimensions, 'lab' for labels, 'dat' for data, and 'des' for design; apart from the parameter values for each command, the only varying commands are 'mod' and 'des' for different models.]

A $\chi^{2}$ value of 836 for 16 degrees of freedom rejects the hypothesis of independence and confirms that there is a systematic relationship between statuses at the two time points. For each status and for each time point, $l E M$ produces parameters that denote the log-odds of being in a status; one can also interpret them in terms of odds. Since we have rejected the independence model, interpreting these parameters is not useful, but we shall do so for the sake of illustration. Thus, for example, for the year 1991, the odds of being in a full-time job is 6.5 times greater than the odds of being in the last reference category 'other.' By 1993, however, these odds declined slightly to 6.1. Similar interpretations can be given for other parameters. Overall, we find that with the same reference group, the most pronounced change in 
Table 2

lEM Commands for Test of Independence Assumption and Log-Linear Estimates

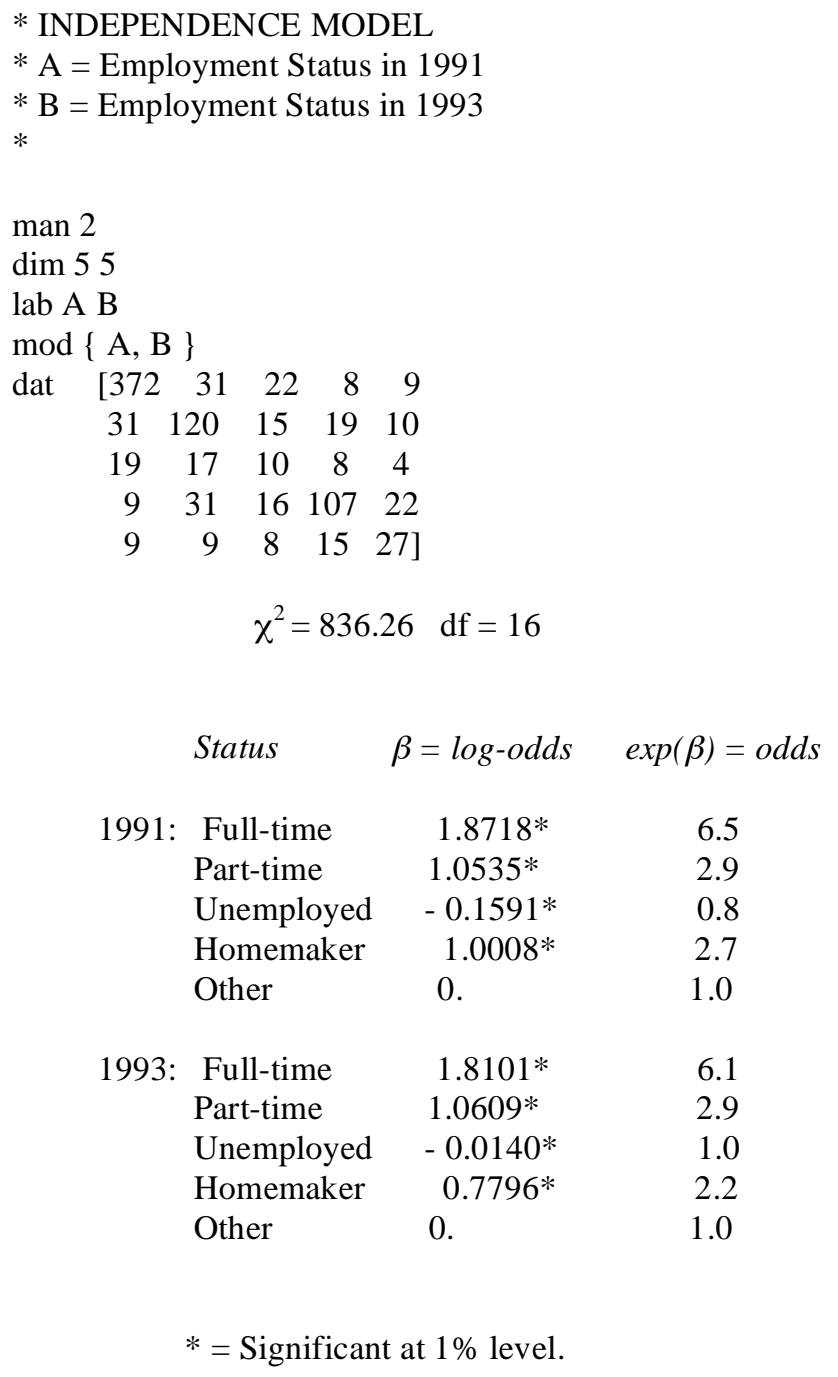


Empirical Research and Applications -

Fernando Rajulton and Zenaida Ravanera

employment status between 1991 and 1993 occurred among homemakers. In 1991 the odds of being a homemaker was 1.25 times greater than in 1993 (2.72/2.18).

Step 2: Once the hypothesis of independence is rejected, we can go ahead for testing the so-called quasi-independence hypothesis. This hypothesis focuses only on those who change their status between the two time points. The quasiindependence model is a variant of the independence model, restricting the analysis only to the frequencies that are off the main diagonal (as if structural zeros occupy the main diagonal). Thus we test the hypothesis that there is no systematic change (as distinct from the relationship implied in the independence model) from time 1 to time 2 .

As seen in Table 3, the quasi-independence hypothesis must also be rejected $\left(\chi^{2}=\right.$ $39.47, \mathrm{df}=11)$. The large decline in the value of the $\chi^{2}$ statistic when the five cells of the main diagonal are set to zero suggests that the quasi-independence model offers a much better fit to the data than the independence model. The parameter estimates given in the table should now be interpreted in terms of 'changing' from and to, and not merely in terms of 'being' as in the independence model. The parameters associated with 1991 point to the odds of changing from the specific job status and those of 1993 point to the odds of changing into the specific status. For example, among the changers, the odds of moving from the 'homemaker' category (1.98) are almost twice the odds of moving from the 'other' category. The 1991 parameter estimates suggest that the changes are most likely to originate from the 'part-time' category and least likely to originate from the 'other' category. Similarly, the 1993 estimates suggest that the changes are most likely to end in the 'part-time' category.

If the quasi-independence hypothesis is rejected, then we can further turn to hypotheses related to systematic change. Here come the symmetry or quasisymmetry models. The former hypothesizes that all changes between 1991 and 1993 are completely balanced. Under this hypothesis, the expected values on the main diagonal will be the same as the observed values (that is, $E_{i i}=f_{i i}$, while the expected values of the off-diagonal elements will be the average of the observed values in the two cells symmetric to the diagonal (that is, $E_{i j}=0.5 *\left(f_{i j}+f_{j i}\right)$. In addition, the symmetry model also assumes the so-called marginal homogeneity, which implies that the marginal distributions do not change because changes from one job status to another will be offset by equal changes from the latter to the former. In other words, the symmetry model implies that no status experiences a net gain or loss between the two time periods.

Table 4 presents the log-linear results of the symmetry model. The model fits the data well ( $p=0.47)$ with a significant improvement over the quasi-independence model. Therefore, the symmetry hypothesis cannot be rejected, and we make the following conclusions. 1) None of the job categories experience a gain or loss from 1991 to 1993 (marginal homogeneity). That is, the proportion of women in each of 
Stability and Change: Illustrations with Categorical and Binary Responses

Table 3

lEM Commands for Test of Quasi-Independence Assumption and Log-Linear Estimates

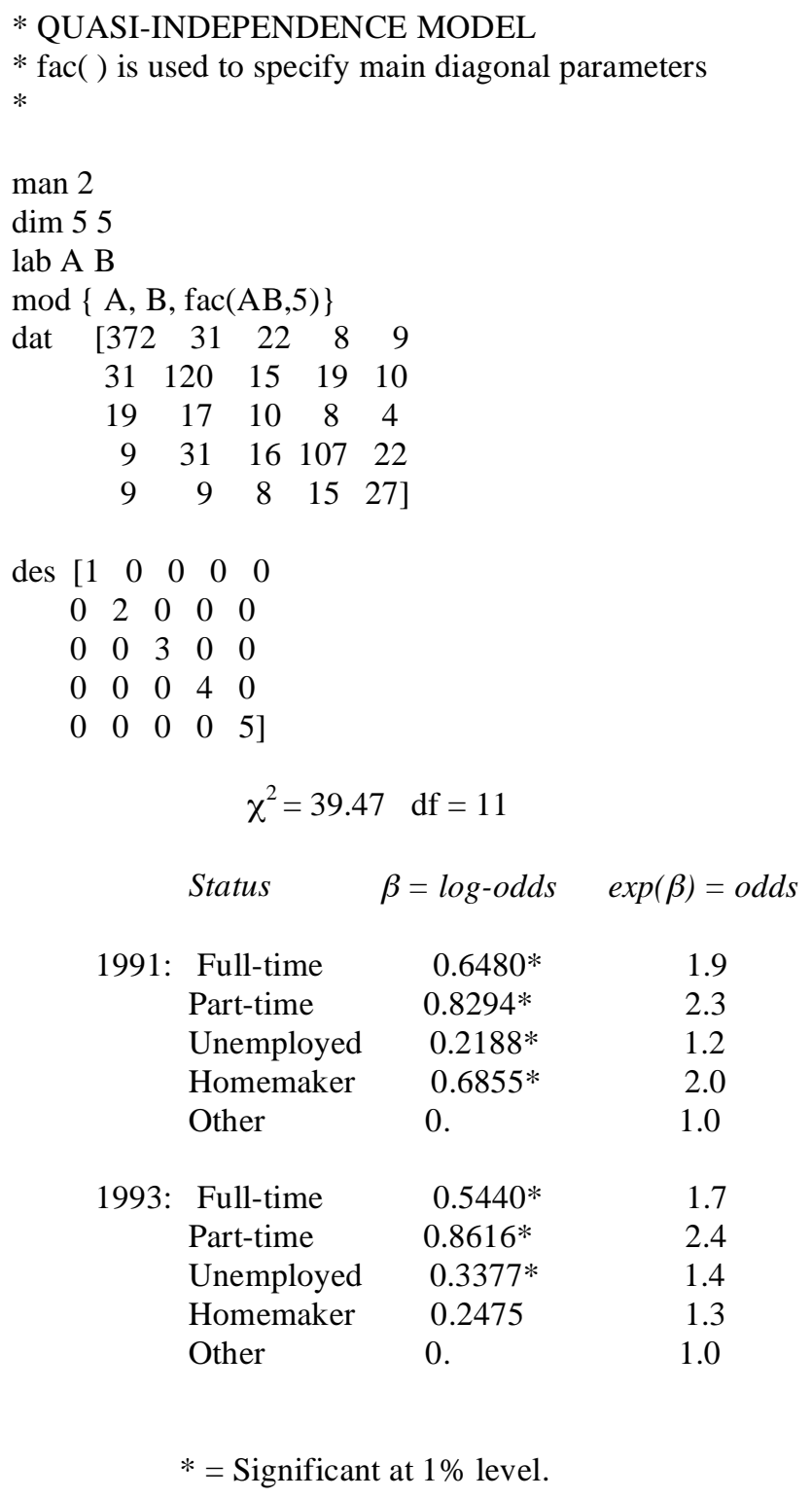


Empirical Research and Applications -

Fernando Rajulton and Zenaida Ravanera

Table 4

lEM Commands for Test of Symmetry Assumption and Log-Linear Estimates

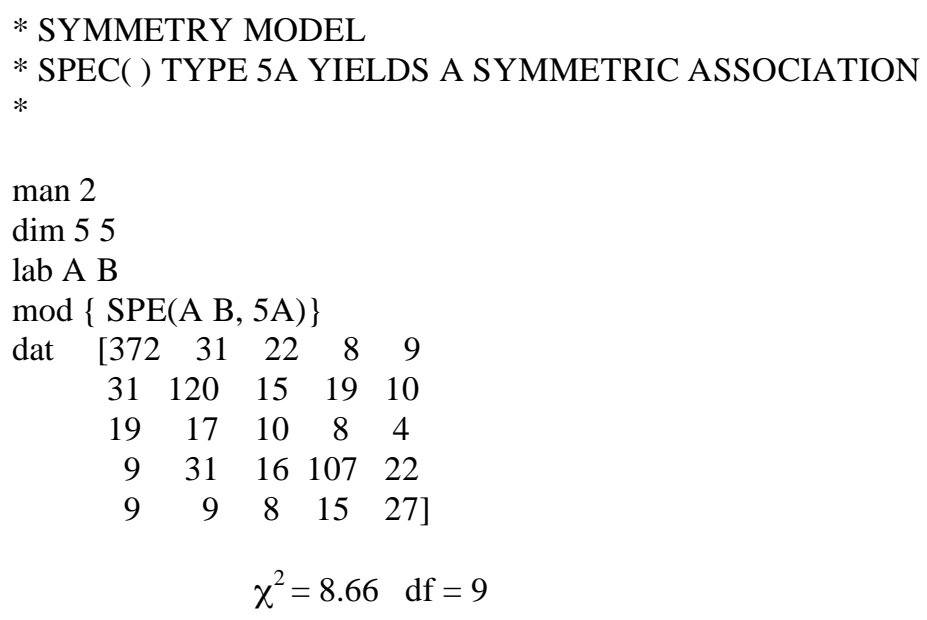

Odds:

1993

$\begin{array}{cccccc} & \mathrm{F} & \mathrm{P} & \mathrm{U} & \mathrm{H} & \mathrm{O} \\ \text { 1991: } & 6.26 & 0.52 & 0.35 & 0.14 & 0.15 \\ \mathrm{P} & & 2.02 & 0.27 & 0.42 & 0.16 \\ \mathrm{U} & & & 0.17 & 0.20 & 0.10 \\ \mathrm{H} & & & & 1.80 & 0.31 \\ \mathrm{O} & & & & & 0.45\end{array}$

All estimates are significant at $1 \%$ level. 
Stability and Change: Illustrations with Categorical and Binary Responses

the five job statuses remained constant from 1991 to 1993. 2) Changes from one status to another were balanced by the changes in the other direction. The parameter estimates given on the main diagonal of Table 4 show the odds of keeping the same job status, and those in the off-diagonal show the odds of changing across the main diagonal. For example, the odds of a woman having a full-time job in 1991 and 1993 is about 3 times higher than the odds of having a part-time job at both time points. Similarly, the odds of a woman having a part-time job in 1991 and being a homemaker in 1993 is 0.42 . Note that according to the symmetry assumption, this is the same as the odds of being a homemaker in 1991 and being a part-timer in 1993. This in turn is 1.5 times more likely than a parttimer in 1991 becoming unemployed in 1993.

What do all these analyses tell us then? Essentially, there is stability in the distributions of the status categories from 1991 to 1993. It is not surprising given a two-year interval between the two waves. The marginal distributions (as found in Table 1) show this stability, that is, the proportions of women in each category have not changed much from one time to the next. This picture of stability is what one would obtain if cross-sectional information were analysed. And yet, we see changes taking place between the two time points, particularly from the part-time and homemaker categories. These changes from and to are significant, according to Table 3. Thus, we are able to get a picture of both stability and change from these log-linear models. If data from more than two waves are available, then we can also examine the patterns of change and stability between time points 1 and 3 , or between 2 and 3 .

If the symmetry were rejected, then we could go for quasi-symmetry model that drops the marginal homogeneity assumption but retains the cancel effect. In the present case, it is not necessary to do so. Interested readers can find a treatment of all these models in some familiar books for analyzing categorical data such as Bishop et al. (1975), Andersen (1990), McCutcheon (1996) and Vermunt (1996, 1997).

\section{Analysing Binary Sequences for Heterogeneity}

When characteristics are measured in binary form from wave to wave, we have binary sequences or recurrent events (e.g., whether employed or not, distressed or not). These binary sequences as dependent variables are of interest here. As with a simple binary dependent variable, when we have several observed covariates, we can fit a standard logistic model (known as fixed effects model) using standard statistical packages. But then we would have to ignore the information on repeated measures. Ignoring repeated measures not only would defeat the purpose of collecting longitudinal data but also would ignore a host of other problems that naturally arise in longitudinal analysis. This particular illustration has the purpose of highlighting the problem of unobserved heterogeneity (or mixture models 
Empirical Research and Applications -

Fernando Rajulton and Zenaida Ravanera

containing both fixed and random effects) in the context of binary sequences. This specific problem has been overemphasized in recent literature despite the obvious conclusion that there is no adequate and satisfactory solution to this specific problem except a sound theoretical and analytical framework at the start. This specific illustration shows how even a simple but sound framework makes unnecessary the complex procedures suggested in the literature about the problem of unobserved heterogeneity.

The package SABRE ( Software for the Analysis of Binary Recurrent Events), written by Dave Stott and freely available on the web (at http://www.cas.lancs.ac.uk/software/sabre3.1/sabre.html), is used for this illustration. This package has been chosen for its simplicity as well as its power of analysis. SABRE can be used to examine the effect of a simple heterogeneity term and can use mass-points. It allows building a mixture model of heterogeneity (in its present version it allows only logistic-normal) as well as other interesting models like Mover-Stayer model, lagged response model, and simple Markov model for two states. For lack of accessibility to micro data files over different waves of longitudinal surveys being conducted in Canada, the data for this illustration are from the example data set (wemp.dat) provided with the SABRE manual. This data set also has the advantage of having as many as ten to 14 repeated measures.

With this data set, we look at the relationship between the employment status and a set of explanatory variables for 155 married women. For each woman, yearly data are recorded, with a total of 1580 measurements (i.e. varying number of records for each woman). The variables in the data file are (see Table 5 for an insert from this data file):

1) $\mathrm{CASE}=$ individual identifier

2) FEMP = wife's employment status; $1=$ employed, $0=$ unemployed

3) $\mathrm{MUNE}=$ husband's employment status; $1=$ unemployed, $0=$ employed

4) TIME = calendar time (year-1975)

5) $\mathrm{UND} 1=$ children aged $<1$ year old; $1=$ yes, $0=$ no

6) UND5 $=$ children aged $<5$ years old; $1=$ yes, $0=$ no

7) $\mathrm{AGE}=$ age (years-35)

As seen from the insert (Table 5), the first woman has four records, the second woman has 14 , the third woman has 8 records.

First, we fit a standard logistic regression to examine the probability of a woman being employed, given the individual characteristics: whether the husband is employed and whether the woman has a child under 5 years. Table 6 displays the model and the parameter estimates. As the results show, if a husband is unemployed, the woman is also less likely to be employed, and the presence of children under 5 lowers the chance of a woman's employment. Both the covariates are highly significant. As usual, we can use these estimates to find the probabilities of a woman being employed, given the specific characteristics. 
Table 5

Insert from the Data File wemp.dat from the SABRE Package

\begin{tabular}{rrrrrrr}
\hline & & & & & & \\
1.0 & 1.0 & 0.0 & 9.0 & 1.0 & 1.0 & -12.0 \\
1.0 & 0.0 & 0.0 & 10.0 & 0.0 & 1.0 & -11.0 \\
1.0 & 0.0 & 0.0 & 11.0 & 0.0 & 1.0 & -10.0 \\
1.0 & 0.0 & 0.0 & 12.0 & 0.0 & 1.0 & -9.0 \\
6.0 & 1.0 & 0.0 & -1.0 & 0.0 & 0.0 & 7.0 \\
6.0 & 1.0 & 0.0 & 0.0 & 0.0 & 0.0 & 8.0 \\
6.0 & 0.0 & 0.0 & 1.0 & 0.0 & 0.0 & 9.0 \\
6.0 & 0.0 & 0.0 & 2.0 & 0.0 & 0.0 & 10.0 \\
6.0 & 1.0 & 0.0 & 3.0 & 0.0 & 0.0 & 11.0 \\
6.0 & 1.0 & 0.0 & 4.0 & 0.0 & 0.0 & 12.0 \\
6.0 & 1.0 & 0.0 & 5.0 & 0.0 & 0.0 & 13.0 \\
6.0 & 1.0 & 0.0 & 6.0 & 0.0 & 0.0 & 14.0 \\
6.0 & 1.0 & 0.0 & 7.0 & 0.0 & 0.0 & 15.0 \\
6.0 & 1.0 & 0.0 & 8.0 & 0.0 & 0.0 & 16.0 \\
6.0 & 1.0 & 0.0 & 9.0 & 0.0 & 0.0 & 17.0 \\
6.0 & 1.0 & 0.0 & 10.0 & 0.0 & 0.0 & 18.0 \\
6.0 & 1.0 & 0.0 & 11.0 & 0.0 & 0.0 & 19.0 \\
6.0 & 1.0 & 0.0 & 12.0 & 0.0 & 0.0 & 20.0 \\
20.0 & 1.0 & 0.0 & 5.0 & 0.0 & 0.0 & -11.0 \\
20.0 & 1.0 & 0.0 & 6.0 & 0.0 & 0.0 & -10.0 \\
20.0 & 1.0 & 0.0 & 7.0 & 0.0 & 0.0 & -9.0 \\
20.0 & 0.0 & 0.0 & 8.0 & 1.0 & 1.0 & -8.0 \\
20.0 & 1.0 & 0.0 & 9.0 & 0.0 & 1.0 & -7.0 \\
20.0 & 1.0 & 0.0 & 10.0 & 0.0 & 1.0 & -6.0 \\
20.0 & 1.0 & 0.0 & 11.0 & 0.0 & 1.0 & -5.0 \\
20.0 & 1.0 & 0.0 & 12.0 & 1.0 & 1.0 & -4.0 \\
& & & & & & \\
& & & & & & \\
\hline & & & & & & \\
\hline
\end{tabular}


Empirical Research and Applications -

Fernando Rajulton and Zenaida Ravanera

\section{Table 6}

\section{Logistic Regression Results for Women's Employment}

Deviance $=1757.36$ d.f. $=1577$

\begin{tabular}{lll}
\hline Parameter & Estimate & Standard Error \\
\hline int & 1.3068 & 0.074415 \\
fmune (1) & 0. & ALIASID [I] \\
fmune (2) & -1.7033 & 0.23584 \\
fund5 (1) & 0. & ALIASID [I] \\
fund5 (2) & -1.7335 & 0.12219 \\
\hline
\end{tabular}

Table 7

\section{Logistic-Normal Mixture Model for Women's Employment}

Deviance $=1237.1568$ d.f. $=1574$

\begin{tabular}{lll}
\hline Parameter & Estimate & Standard Error \\
\hline int & 2.0098 & $0 . .19833$ \\
fmune (1) & 0. & ALIASID [I] \\
fmune (2) & -2.7033 & 0.42577 \\
fund5 (1) & 0. & ALIASID [I] \\
fund5 (2) & -2.6619 & 0.23273 \\
scale & 2.1082 & 0.19258 \\
\hline
\end{tabular}

With the above information from the logistic fit, we move on to introduce randomness by incorporating a mixture model for examining the likelihood of women's employment. SABRE allows only logistic-normal mixture, with endpoints if desired, and the results from this model are displayed in Table 7. Compared with the standard logistic model, this model reduces the $\chi^{2}$ value ('deviance' in the table) by $1757.36-1237.16=520.20$ for 3 degrees of freedom. The parameter estimates are now larger with the heterogeneity term included in the model. In addition, we have the scale parameter (2.1082) that is significant. This 
Stability and Change: Illustrations with Categorical and Binary Responses

scale parameter is the unknown standard deviation of the mixing distribution of heterogeneity. With the assumed normal structure, this value means that $95 \%$ of subjects would fall within 4.2 (that is, $\pm 2 \sigma$ ) logit units of the overall mean. This range on the logit scale translates into probabilities that range from 0 to 0.98 , implying that some women have little chance, while others have high chance, of employment, given the two covariates - a wide spectrum of heterogeneity present in the data.

We can continue in this manner to fit other models that may be theoretically relevant; for example, a lagged logistic-normal mixture that would consider besides a term for heterogeneity the impact of the initial status on the measurements subsequently observed. This model is therefore related to some important aspects of life history analysis, particularly in a non-Markovian-type framework. Table 8 presents the results obtained by fitting this lagged logistic-mixture model to the data, and the results are enlightening. First, the model reduces the deviance value thus making it the best model fit. Second, the parameter estimates are still stable even though lessened in their magnitude, except for the intercept that turns negative now. The lag parameter becomes prominently distinct and significant. The scale parameter is reduced by half (compared to the previous model), even though still significant. Thus, the initial status or the lag parameter becomes a very important variable; it reduces unobserved heterogeneity by $50 \%$. This reveals to us the importance of doing a Markovian or non-Markovian analysis.

\section{Table 8}

\section{Lagged Logistic-Normal Model}

Deviance $=8783.94$

Deviance decrease from the model

Without the lag term $=\mathbf{2 2 0 . 7 2}$ for 1 d.f.

\begin{tabular}{lll}
\hline Parameter & Estimate & Standard Error \\
\hline int & -0.81251 & 0.22417 \\
fmune (1) & 0. & ALIASID [I] \\
fmune (2) & -1.6864 & 0.41477 \\
fund5 (1) & 0. & ALIASID [I] \\
fund5 (2) & -1.0872 & 0.23191 \\
lag & 3.5967 & 0.22898 \\
scale & 0.93090 & 0.21221 \\
\hline
\end{tabular}


Empirical Research and Applications -

Fernando Rajulton and Zenaida Ravanera

The package SABRE allows the fitting of Markov logistic-normal mixture model as well. The results from this model are shown in Table 9 where the parameter estimates are classified by the initial status whether employed and unemployed. For women who started their history in unemployed status, having children under 5 is no longer significant and the scale parameter is larger than the one in the lagged model. In contrast, for women who started in employed status, the scale parameter almost disappears and becomes nonsignificant. This interesting analysis clearly shows that unobserved heterogeneity, if any, is entirely because of the group of women who started their history in unemployed status and that including the initial status in the analysis eliminates unnecessary concerns about unobserved heterogeneity. The lesson from all these exercises is clear: Do not neglect past history in longitudinal analysis.

\section{Table 9}

\section{Markov Logistic-Normal Model}

Deviance $=846.49$

$\begin{array}{lll}\text { Parameter } & \text { Estimate } & \text { Standard Error }\end{array}$

\section{1) Initial Status: Unemployed}

$\begin{array}{lll}\text { int } & -1.3981 & 0.2906 \\ \text { fmune (1) } & 0 . & \text { ALIASID [I] } \\ \text { fmune (2) } & -1.99344 & 0.7470 \\ \text { fund5 (1) } & 0 . & \text { ALIASID [I] } \\ \text { fund5 (2) } & -0.2510 & 0.3512 \\ \text { scale } & 1.2878 & 0.2995\end{array}$

\section{1) Initial Status: Employed}

$\begin{array}{lll}\text { int } & 3.0890 & 0.1802 \\ \text { fmune (1) } & 0 . & \text { ALIASID [I] } \\ \text { fmune (2) } & -1.3567 & 0.4653 \\ \text { fund5 (1) } & 0 . & \text { ALIASID [I] } \\ \text { fund5 (2) } & -1.8990 & 0.2643 \\ \text { scale } & 0.1160 & 0.2767\end{array}$


Stability and Change: Illustrations with Categorical and Binary Responses

\section{Conclusions}

In this paper we have discussed and illustrated the following important points about longitudinal analysis. First, longitudinal data provide rich information on stability and change in the behaviour of systems as well as individuals, and these two dimensions should be examined together, not one or the other.

Second, longitudinal data introduce many complexities. One such complexity is that of unobserved heterogeneity that has been much discussed in recent literature. Though its usefulness is under debate, research experience tells us that nothing is more important than a good research design backed by a solid theoretical framework. It is disappointing to read many research papers that use very complicated and sophisticated techniques of analysis and finally make simplistic statements that unobserved heterogeneity is significant and should be considered in the analysis. What is worse, in an attempt to explain unobserved heterogeneity, they include all imaginable attributes under the sun that cannot be easily observed. Researchers have the obligation to show what unobserved heterogeneity found to be significant points to. Our experience shows that inclusion of past history, no matter how indirectly introduced into an analysis, often removes the need for considering the heterogeneity term in an analysis.

Third, a point regarding the distribution that can be used in building a model like the one shown above: If any nonlinear program is available, we strongly recommend the use of beta-logistic distribution in contexts of longitudinal research. With beta-logistic, it is possible to examine repeated measures for "'heterogeneity in persistence.' That is, with information on the same category identified $j$ out of $n$ times (waves), we include a beta distribution to allow for unobservable persistence over time. A case in point is voters' persistence in the elections held in Canada; it is well known that Catholics have been persistently choosing the Liberals in Canada since the beginning of the $20^{\text {th }}$ century.

Fourth, the above illustrations have intentionally dealt with lower-level variables categorical and binary variables -, which many have come to consider as less useful than ratio-level variables. Far from it. Many longitudinal surveys in social research still collect much information in categorical or binary form. And the techniques are as 'sophisticated' as, say, hazard models and can bring to light many hidden gems in longitudinal data.

Fifth, the dynamic analysis of life histories that examines the impact of history is one of the best ways to analyze longitudinal data. The key ideas in life history, namely timing and sequence, are the most suitable to analyzing any longitudinal data, provided proper measurements are used at the time of observation. A separate paper in this Special Issue deals with this topic in detail.

Lastly, longitudinal data are the best sources for examining growth, especially the so-called intra-individual variability. As mentioned earlier, both individuals and 
Empirical Research and Applications -

Fernando Rajulton and Zenaida Ravanera

systems experience stability and change. Empirical analyses, however, pit one against the other as if one of them is all that matters. The main aim of collecting longitudinal data is to identify the growth, development and changes taking place in individuals or groups. Thus, the 'intra' aspect gains more importance in longitudinal analysis, more so with repeated measurements. Individuals differ not only from one another (inter-) but also from one time point to another (intra-). The intra aspect can point to either stable or changing characteristics in individuals themselves, either short-term or long-term. In fact, it is intra-variability that contributes to inter-variability observed among individuals, and yet we do all our analyses focusing on inter-variability that is often confounded with intra-variability. Longitudinal data promise a new focused direction towards examining intraindividual variability.

\section{End Notes:}

1. As Cleveland (1993) showed, statisticians had been using for decades the wellknown barley data obtained through an experiment in the state of Minnesota, the data that have been used since the time of R.A. Fisher for illustrations on randomization and analysis of variance. But they all missed observing one major error in the data, an error that could be detected only with the trellis display.

2. We would recommend to readers a graphic package DATAPLOT that is freely available for downloading on the web page of the National Institute of Standards and Technology. Go to:

http://www.itl.nist.gov/div898/software/dataplot.

3. This example was worked out by Piotr Wilk for a course assignment, and I express my thanks to him for allowing me to use his work for illustration in this paper.

4. EM can be downloaded from the Tilburg University's webpage: http://www.kub.nl/faculteiten/fsw/organisatie/departementen/mto/software2 .html.

\section{References:}

Andersen, E. B. 1990. The Statistical Analysis of Categorical Data. Berlin: Springer-Verlag.

Avison, W. 1996. Mental and physical health of mothers and children in singleparent families, London Ontario. The University of Western Ontario, London Ont., Canada. 
Stability and Change: Illustrations with Categorical and Binary Responses

Bishop, Y.M.M., S.E. Fienberg and P.W. Holland, 1975. Discrete Multivariate Analysis: Theory and Practice. Cambride, Mass.: MIT.

Cain, L. D. Jr, 1964. Life course and social structure, in R.E.L. Faris (ed.), Handbook of Modern Sociology. Chicago:Rand McNally. Pp. 272-309.

Cleveland, W. S., 1993. Visualizing Data. NJ:Hobart Press.

Coale, A. J., 1973. The demographic transition reconsidered. Proceedings of the International Population Conference, Liege. Pp.53-72.

Coleman, J. S., 1990. Foundations of Social Theory. Cambridge MA:Belknap Press of Harvard University.

Elder, G. H., Jr. (1975). “Age differentiation and the life course,” Annual Review of Sociology 1: 165-190.

Engel U. and J. Reinecke (eds), 1996. Analysis of Change, Advanced Techniques in Panel Data Analysis. New York: Walter de Gruyter.

Featherman, D. L. and R. W. Lerner, 1985. "Ontogenesis and sociogenesis: Problematics for theory and research about development and socialization across the lifespan," American Sociological Review 50: 659-676.

Frederiksen, C. H. and J. A. Rotondo, 1979. Time-series models and the study of longitudinal change. In J.R. Nesselroade and P.B. Baltes, Longitudinal Research in the Study of Behavior and Development. NY:Academic Press.

Giele, J. Z. and G. H. Elder Jr., 1998. Methods of Life Course Research, Quantitative and Qualitative Approaches. London:Sage.

Gottman, J. M., 1995. The Analysis of Change. UK: Lawrence Erlbaum.

Haberman, S. J. 1979. Analysis of Qualitative Data. N.Y.:Academic Press.

Jones, R. H. 1993. Longitudinal Data with Serial Correlation: A State-space Approach. N.Y:Chapman \& Hall.

Kohli, M., 1986. The world we forgot: A historical review of the life course, in V.W. Marshall (ed.), Later Life: The Social Psychology of Aging. Beverly Hills CA: Sage. Pp. 271-303. 
Empirical Research and Applications -

Fernando Rajulton and Zenaida Ravanera

Lesthaeghe, R. and C.Vandehoeft, 1998. Ready, willing and able: A conceptualization of transition to new behavioural forms. Paper presented at the National Academy of Sciences Meetings on the ASocial Dynamics of Fertility Change in Developing Countries, Washington DC.

Magnusson, D., L. R. Bergman, G. Rudinger and B. Torestad (eds.), 1991. Problems and Methods in Longitudinal Research: Stability and Change. New York.:Cambridge University Press.

Mannan, H. R., 1999. Contraceptive usage and method choice in Bangladesh. MA thesis, Department of Sociology, University of Western Ontario, London Ontario.

McCutcheon, A. L., 1996. Models for the analysis of categorically scored panel data. In Engel and Reinecke (eds.), 1996.

Menard, S. 1991. Longitudinal Research. London:Sage.

Nesselroade and Featherman, 1991. Intradindividual variability in older adults= depression scores: Some implications for developmental theory and longitudinal research. In Magnusson et al. (Eds), pp. 47 seq.

Reinecke, J. and Schmidt, 1996. Model specification and missing value treatment in panel data: testing the theory of planned behaviour in a three-wave panel study, in Engel and Reinecke (eds.), 1996.

Uncles, M. D. (Ed.), 1988. Longitudinal Data Analysis: Methods and Applications. London Papers in Regional Science, London.

Vermunt, J. K., 1996. Causal log-linear modeling with latent variables and missing data, in Engel and Reinecke (eds.). Pp.35-60.

Vermunt, J.K., 1997. LEM: A general program for the analysis of categorical data. Tilburg University.

Waterton and Lievesley, 1988. Attrition, conditioning and attitude change: Some findings from the Social Attitudes Panel Study, in Uncles (ed.). Pp.7388. 